\title{
Applying Framing Theory in Digital Transformation Research: Suggestions for Future Research
}

\author{
Frida Ivarsson \\ Gothenburg University \\ Swedish Center for Digital Innovation \\ frida.ivarsson@ait.gu.se
}

\begin{abstract}
Meaning-making processes are essential to guide new action pathways in organizations. How organizations engage in meaning making of digital technologies should therefore be of particular interest in digital transformation (DT) research. This study explores how an existing theory of meaning making in organizations, namely, framing theory, can be applied in DT research. It contributes to the literature by offering a synthesis of framing theory, with an emphasis on framing of digital and information technologies in organizational contexts and proposing research questions inspired by framing theory for future DT research.
\end{abstract}

\section{Introduction}

In the digitalizing society, all organizations face a recurrent need to engage in meaning making of the characteristics, affordances, and implications of digital technologies for organizational life. The digital environment is rife with tech-savvy concepts that represent emerging digital technologies (e.g., artificial intelligence, blockchain, digital platforms, quantum computing) -all examples of powerful and complex digital technologies with major implications for organizing [54]. Understanding the promises and consequences of digital technology may even be pivotal to the long-term prosperity of an organization (e.g., Kodak) [31]. Therefore, how meanings of digital technologies are formed, adapted, disputed, shifted, and diffused and the role of such meaning-making processes in guiding digital technology development and organizing make a ripe research topic to explore [33].

This study explores how an existing theory of meaning making in organizations can address this topic. The theory of choice is framing theory. Broadly, framing theory describes and explains the dynamic, social, and oftentimes contested processes where actors "(re)think," (re)interpret, or (re)shape meanings of technologies in a particular organizational context, including their efforts to influence others' meaning making of technologies.

To position this inquiry in a relevant and timely area of concern, I ask the following question: How can framing theory be useful for digital transformation research? Meaning making of digital technologies in organizations should be a particularly attractive topic to explore in digital transformation (DT) research, as it is an area of concern with a broadly defined interest in "organizational change that is triggered and shaped by the widespread diffusion of digital technologies" [18]. At the same time, framing theory has the potential to be useful for DT research as it is applied similarly in research on organizational change [9], building on the assumption that the meanings actors ascribe to "the world" must change for significant change in action to occur [10]. Hence, the objective of this study is to suggest research questions inspired by framing theory for future DT research.

To answer this question, this study presents a literature review of framing theory in information systems (IS) research and discusses how the theory can be applied in DT research. I begin the paper by introducing framing theory in more detail. Next, I define and outline the view on DT that underlies the research and connect DT to the notion of framing. I continue by describing the methodological approach of the literature review. Thereafter, I present a synthesis of framing theory, organized into six subareas. Lastly, I propose research questions inspired by framing theory for future DT research in the Discussion.

\section{Framing Theory}

The essence of framing theory is the "construction of meaning," where meanings are formed by a reciprocal and recursive relationship between discourse and cognition [9]. Goffman, who is typically attributed for popularizing the concept, defines frames as 
"schemata[s] of interpretation." In this view, frames refer to simplified cognitive representations of reality that guide actors in understanding the world and acting in it [17]. The notion was extended to IS research when the notion technological frames of reference (TFR) was introduced, referring to "the core set of assumptions, expectations, and knowledge of technology collectively held by a group or community." [36]. While this definition suggests a socio-cognitive perspective on frames, it is criticized for being technology-centric without acknowledgement of context, lacking explanatory power of temporal change in frames and an incomplete acknowledgement of the social processes that shape frames [14]. Along similar lines, other scholars have called for an "increasing emphasis on framing as a dynamic process and on the characteristics of this process" [10], that views "frame change as an ongoing interpretative process, triggered by a variety of organizational circumstances". As this paper will demonstrate, framing theorizing in IS research has since come to take a broader approach than the initial TFR theory. I now continue with defining DT, and connecting it to the notion of framing.

\section{Digital Transformation}

Digital transformation (DT) has emerged as a distinct area of concern with a broad interest in organizational change, stemming from the changing conditions for organizing triggered and shaped by digital technologies. As the area is nascent, a number of struggles prevail in the DT literature. Such struggles include differences in viewing the outcome of DT as a state of malleable organizational designs [18] or a new organizational identity [50] versus viewing DT as a continuous process [45]; applying the DT concept to a broad range of entities (e.g., ecosystems, industries, societies) [42] or practices [34] versus applying the concept restrictively to organizations [18]; and the different degrees in emphasis on actors' intentionality [42] versus responsiveness to unforeseen events during DT [18]. Therefore, consensus on how to define DT is still lacking [26], which creates a need to outline the view that underlies DT discussions.

In this study, I view DT as a malleable form of organizing that enables continuous adaptation for influential digital ecosystem orchestration. In this view, organizing constitutes the organization because change is continuous [48]. Furthermore, it views organizing as situated in ecosystems [18], referring to networks of people, organizations, and artifacts that potentially span traditional industry boundaries. In other words, organizing is situated in a dynamic space of actors and artifacts in a relational network, thereby making ecosystems "agent-artifact space[s]" [27].
Digital technologies establish new ecosystem characteristics. Digital ecosystems are distinct from traditional inter-organizational networks in that they enable an increased scale and diversity of participating actors, and a continuously emergent ecosystem structure given the malleability of digital technologies and the generativity they enable [23, 44, 54]. Operating in digital ecosystems makes organizations inherently externally oriented and boundary spanning. Maintaining an influential position in the ecosystem requires subtle coordination mechanisms, as organizations engage and guide distributed and largely autonomous actors in economic and social activities enabled by digital technologies (i.e., digital ecosystem orchestration) [23]. Digital ecosystem orchestration is thus the distinct locus of intentionality associated with DT in this view (cf. ITenabled org. change [50]).

To maintain an influential position in digital ecosystems, which are highly dynamic environments, organizations must both proactively initiate change and reactively respond to externally initiated changes [48]. This is because actors can generate digital technologies themselves (e.g., establishing a platform), contribute to the development of technologies that other ecosystem actors generate (i.e., as a platform complementor of applications and content), and/or coordinate other ecosystem actors' generation of applications and content around proprietary digital technologies (i.e., inviting complementors as a platform owner) [8].

For organizations, digital ecosystems are therefore associated with a lack of long-term predictability of pathways and outcomes because conditions repeatedly and rapidly change [12]. Operating in an environment of continuous change requires a malleable form of organizing [18], where organizational arrangements are easily modifiable to enable intentional and responsive actions for sustained or improved digital ecosystem orchestration [18,23]. Malleable organizing thus entails the capacity to quickly and effortlessly reconfigure organizational arrangements within the boundaries of established organizing principles, for instance, "adding, splitting, transferring, merging or deleting of units without change to fundamental structural principles" [16].

In the context of continuous change-and DT in particular - organizational life is recurrently punctuated by the need to engage in meaning making. When sensing new affordances of emerging digital technologies for digital ecosystem orchestration or sensing changes in the ecosystem, organizations need to "recognize adaptive emergent changes, make them more salient, and reframe them" [48] in order to act in alternative directions [10]. The need is on-going, given that new digital technologies continuingly emerge and established ones are potentially ever-changing [44, 54]. 
Although extant research approaches DT from various theoretical perspectives, including institutional theory [21], dynamic capabilities [30, 42, 45], strategizing [7], paradoxical tensions [51] and organizational identity [50], these are insufficient to explain the role of meaning making in DT. Therefore, I now continue with a literature review of framing theory as one potential theoretical option to discuss how it can be applied in DT research.

\section{Literature Review Approach}

The intention with this literature review was to synthesize framing theorizing in IS research into themes that could guide a discussion of future DT research. Therefore, I chose to perform an organizing review, which is a type of literature review that focuses on description and synthesis [28]. Given this objective, the sampling process aimed to collect a representative sample of papers with significant theoretical contributions [43]. The search process had the following boundaries for filtering papers of relevance: (1) papers published from 2006 and onwards, which was when the call for an increased focus on framing as process in IS research was published [10]; (2) papers with a theoretical perspective that acknowledges change in frames and/or organizations [6]; (3) papers concerned with framing of an IT or digital artifact or framing more broadly during the development, implementation, use, (re)design or similar of such artifact; and (4) papers that situate framing in the context of organizations.

I used Web of Science to search for papers. The process involved three steps (Image 1). It started with a search of leading journals (step 1), followed by a backward search to identify prior publications through searching the reference lists of papers from step 1 (step 2 ), and a forward search to identify later publications that papers from step 1 cite (step 3) [46]. To maintain consistency in rigour across the steps, I excluded conference papers and papers published in journals with an impact factor $<2.6$ in 2019 , which was determined based on the lowest impact factors amongst the selected leading journals.

For step 1, I started by searching the AIS Senior Scholars' Basket of Journals ${ }^{1}$. I used a variety of keyword strings. These were iteratively developed, and resulted in eight variations of the words "fram*," "technolog*," "organiz*," "digital," "IT," "chang*," and "innovation". For example, I added the keyword "chang*" later on because some papers discussed change in frames rather than framing. Asterisks were added to ensure that variations in conjugations of words were included (e.g., “organization(s)," “organizing," "organizational," etc.). Step 1 was conducted on April 7,2021 , and rendered a total of 105 papers. I did manual filtering by reading through the abstracts and identified many papers as irrelevant because "framing" and "frames" are words with multiple meanings in the context of research, such as outlining the conceptual or theoretical "framing" of a paper. This resulted in a sharp reduction to 12 publications. For step 2, the backward search was conducted by manually scanning the citation lists of the papers from step 1, resulting in an accumulation of 18 papers in total. Finally, for step 3, forward search was done by manually scanning abstracts of papers identified through Web of Science, rendering a sample of 26 papers. These 26 papers were read in depth for a final filtering of papers before proceeding to analysis, resulting in a final sample of 14 papers (Table 1). This last in-depth reading was needed because it revealed that framing was not the main theoretical device used in all papers, though it was used as a descriptive concept in the abstract of some.

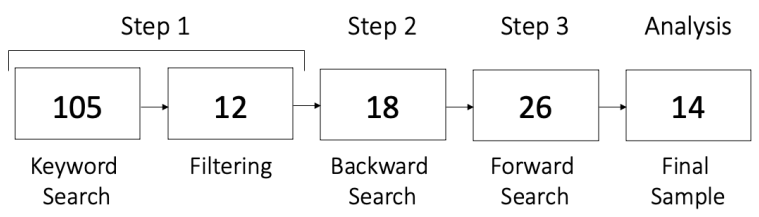

Image 1. Overview of the search process.

In the analysis, I summarized each paper and engaged in two rounds of coding using Atlas. TI coding software. Throughout the coding, I carefully considered what level(s) of analysis the papers were concerned with and how levels interact in some instances. Here, I coded the data by using the distinctions macro (field level), meso (organizational or group level) and micro (individual) levels, as used in framing research in organization studies [9]. In the first coding round, I coded the final sample of papers openly, with the intent to stay close to the words and claims used by the authors. I also coded what kinds of relationships exist between the first order codes as outlined by the authors in their theorizing. For example, "frame evolution" and "frame contestation" were given the relation "is a form of" to "political contest." In turn, the codes "apply[ing] organizational power," "power balance between actors," "interests," and "belief in options" had the relation "shapes" to the code "political contests."

In the second round of coding, I constructed a coding network to identify clusters amongst codes and their relations. The relations between first-order codes formed the basis for grouping codes together. For

${ }^{1}$ AIS Senior Scholars' Basket of Journals (n.d.) 
example, codes related to the code "political contest" through the relation "shape" were outlined together, along with their subcodes (e.g., related codes through "is part of," "can manifest as," etc.). As clusters emerged in the code network, I identified and labeled second-order themes [5]. For example, the second-order code "power" was given to codes mentioned above as "shap[ing]" "political contests." Next, I added overarching codes to group second-order themes together. For example, the second-order codes "power," "culture," and "history" were coded as "contextual conditions" and linked through the relation "is a." These overarching codes represent what I describe as subareas in framing theory. I now proceed to the synthesis.

\section{Synthesis}

In this section, I present framing theory across 6 subareas, namely, relational framing, political framing, tactical framing, technological framing, temporal conditions and contextual conditions. The first four subareas represent characteristics of framing processes, and the last two represent conditions that shape framing processes. "Actor" can refer to an individual, a group of individuals, an organizational unit, or an organization, depending on the level of analysis. I specify where the text concerns actors on specific levels of analysis. An overview of the contribution and level(s) of analysis of each paper is found in a concept matrix (Table 1) [46].

\subsection{Relational Framing}

Framing processes are always relational, because interactions drive the generation of new or alternative meanings of technologies in organizations. A number of distinct interactional patterns can be distinguished in relational framing. One interactional pattern highlights the relational framing between different actors, for instance, between organizations [4, 22]; occupational groups or departments in organizations [20, 29, 35, 52]; designers and users [2]; or organizational members situated in different cultural contexts [38]. Such relational framing focuses on resolving differences in meanings held by diverse actors of a particular technology, referred to as frame incongruences [55].

A second interactional pattern concerns relational framing within actor constellations (e.g., groups, individuals). Similarly, such relational framing is concerned with resolving differences in frames, but it is distinctly referred to as frame inconsistencies [55]. If unresolved, frame inconsistencies can lead to contradictory actions [55]. For example, frame inconsistencies can manifest as frame ambiguity, where actors leave open the possibility for more than one interpretation, or frame switching, where actors draw on more than one frame in one instance (e.g., in a written report) [20]. Frame inconsistencies also interact with frame incongruences and how they do shape framing outcomes, such as by negatively or positively reinforcing one another depending on actions taken to resolve situations of incongruence/inconsistency [55].

Third, framing theory highlights interactional patterns in relational framing across different levels. For example, it is suggested that interactions between diverse micro- and meso-level actors with differing frames (users, producers, institutions) may result in a macro-level frame, specifically, a dominant technological design [24]. In turn, this macro-level frame has the potential to shape a technology trajectory across an entire industry if conflicting differences are resolved. Moreover, an established organizational frame can have a negative impact on individual actors' reframing efforts of technologies [25]. While individuals may temporarily change how they envision technological potential in their organizations, they may return to interpreting technologies in light of the dominant organizational frame as they return to their everyday operations [25].

\subsection{Political Framing}

Political framing manifests in framing if diverse actors have differing subjective interests in a particular technology that they share prospects in [52] or adhere to different values that are difficult to unite within a shared frame [4, 38]. For example, political framing can involve disputes over what functionality a particular technology should have and why, or what/whose purposes it should serve. It highlights the role of contests around what frame should be dominant in an organization ("dominant frame") [35], that is, a frame that prevails over alternative frames at a specific point in time. If some actors do not accept a dominant frame, then contests can manifest as conflict or resistance [35].

Actors can engage in political framing in different ways. On the one hand, political framing can drive actors to mobilize support amongst "bystanders" or advocates of a similar view [2] to influence change in others' frames in some particular direction [4, 22]. This approach represents an attempt to consolidate power [4]. On the other hand, political framing can drive actors to try to extend one's own understanding of others' frames in an attempt to avoid conflict [38]. To exemplify the latter, actors can use cultural resources (subjective beliefs about other cultures, in this paper) to extend their own frames. However, this approach risks reproducing stereotypical assumptions about others [ibid.]. 


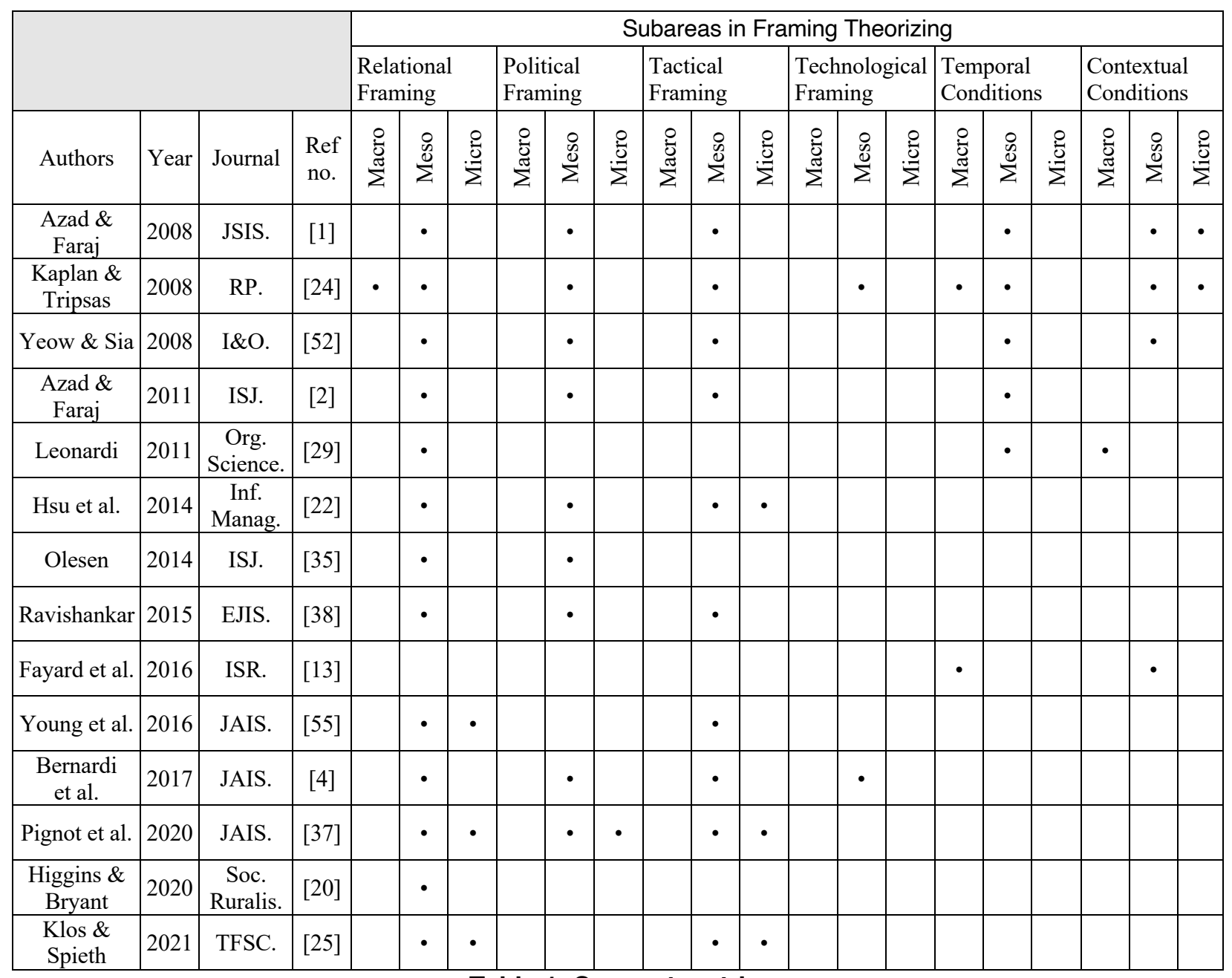

Table 1. Concept matrix.

\subsection{Tactical Framing}

Tactical framing refers to goal-oriented acts that actors deploy with the intention to invoke change in others' frames in some particular direction (e.g., diffusing, developing, motivating, legitimating frames etc.) [4]. Framing theory acknowledges two forms of tactical framing. The first is tactical framing that includes rhetorical acts, referring to "the instrumental use of persuasive language and discourses" [22]. Here, actors make use of "discursive devices" when interacting with others, such as stories, metaphors, visual images, appeals to principle, representative examples, and catchphrases [2]. Additionally, actors need to selectively consider the structure of rhetorical acts, more closely the pattern and content of arguments, to appeal to a specific audience. For example, they can choose to appeal to others' cultural values in seeking to mobilize others [52]. They can also include affectivelyladen discourse (i.e., appeal to ideology), where actors seek to appeal to the identification and belonging needs of individuals; in this paper, reframing a game engine as an alternative to other proprietary game engines by using notions such as democratization, citizenship, and heroism in rhetorical acts, as a way to exploit others' vulnerabilities from the authors' critical stance [37]. Rhetorical acts are situational, as choices in what rhetorical acts to use may need to be reconsidered throughout the framing process to achieve a particular goal. For example, changing the choice of words and rethinking who conveys the message can improve frame congruence [22]. However, it can be strategic to hold on to interests and values in rhetorical acts in some instances, such as for less influential actors who contest a dominant frame [4].

The second form is tactical framing that includes organizing acts, which incorporate decisions about how interactional spaces are designed (e.g., participants, meeting structure, distribution of voice, content in agendas, etc.). The purpose of organizing acts can be 
directly targeted to (re)shape meanings of technologies (e.g., on what characterizes a technology and what it could afford for a particular organization). For example, organizing for such purpose can involve foresight activities, referring to future-oriented activities where "organizational members are confronted with specific situations and technologies that require them to apply and adjust their individual frames to make sense for the future development of their industry and organization" (i.e., scenario building) [25]. Institutions (e.g., standard setting bodies) can play a significant role in organizing cross-boundary framing activities of emerging technologies [24].

Organizing acts can serve other purposes as well. They can also be designed as negotiations that focus on reconciling emerging contests between actors during framing $[1,52]$. The potential emergence of frame incongruences and frame inconsistencies may likewise surface the need to organize instances of framing anew [55]. The present study proposes designing different interactional spaces for these distinct types of situations. On the one hand, attempts to reconcile frame incongruences require discussions focused on perspective taking where actors "engage in appreciating the perspectives of other groups". On the other hand, frame inconsistencies require perspective making where the goal is to "identify, examine, and adjust assumptions to reduce contradictions" [55]. Thus, the choices of organizing acts may need to be reconsidered over time similarly as rhetorical acts, depending on how the framing process unfolds.

\subsection{Technological Framing}

Framing theory acknowledges that technologies play an active part in framing processes. Specifically, it accentuates that materialized technologies provide continuity to frames [4]. This is because technologies mediate and diffuse actors' frames because past design decisions become inscribed in them. In this sense, materialized technologies become means for sustaining frames in organizations over time and space [ibid], for instance, through the inscription of actors' values and interests in technology design [2]. Furthermore, framing theory recognizes that the artifactual characteristics and functionalities of technologies limit what interpretations are possible [24]. If actors find that a particular technology cannot perform within the boundaries of one frame during development, this can trigger the need for reframing. To illustrate the principle with a "non-digital" example, a pharmaceutical firm generated a reframing of a biotechnology, from a technology that "create[s] large molecule drugs" to "a research method for locating small molecule drugs" when it could not deliver as initially intended [24].

\subsection{Temporal Conditions}

Temporal conditions concern changes in the character of framing over time in organizations. Essentially, framing theory suggests that framing differs in significance and form across different phases in relation to technological change, in relation to both macro-level (e.g., industry-shared understandings of technologies throughout technology life cycles [24]) and a specific technology application. In relation to macro-level frames, framing has different influence throughout the technology life cycle [24]. During "the era of ferment" when new technologies emerge and variety between different frames is particularly high, framing is especially salient and situational in organizations that are early to sense emerging technologies [13]. Framing is also salient in the selection phase, which follows the era of ferment, where a dominant design may emerge through eventual negotiations between actors' competing frames [24]. This phase is defined by a greater scale and diversity of participating actors in framing. Finally, framing manifests in the era of technological discontinuity, where, for example, start-ups and users whose frames may not be bound by an industry-shared frame discover emerging technologies and possibly engage in challenging existing frames [24].

In framing particular technology applications in organizations, it is suggested that framing differs across different phases of development. For example, the conceptual development phase is distinguished from the technology implementation phase through how cultural resources are activated in these phases [29]. While culture shapes how technologies are framed by actors in the implementation phase, technology concepts (or "ideas" about technology) are already activating cultural resources in the conceptual development phase. This means that different actors use different cultural frames to interpret the problem that technological artifacts are eventually intended to solve [29]. Framing efforts may also continue during a post-implementation phase, such as framing efforts to sustain the dominant frame [52]. Finally, framing theory suggests that different phases in framing contests $[1,2]$ characterize framing processes if situations of political contests unfold, including distinct phases in how contests are reconciled [1] and how power manifests during contests [2].

\subsection{Contextual Conditions}

Framing processes are shaped by the contextual conditions of an organization. The contextual conditions highlighted in the sample are, broadly, history, power balances between actors and culture. History shapes framing processes, because pre-existing organizational 
frames incorporate "the encoding of their prior history, including both idiosyncratic organizational experiences and industry affiliations" [24], which actors depart from in interpreting new experiences. The impact of history manifests as, for example, how the past and present belonging to a specific industry or sector constitutes a point of departure for actors' reframing efforts. It also manifests in micro-level framing, given that individuals bring frames encoded by their prior experiences into new organizational contexts [24]. Therefore, history is a condition that shapes framing in both incumbent and newly formed organization (e.g., start-up firms).

The prevailing power balance between different actors creates a second contextual condition that shapes framing processes [1]. For example, actors in established positions of power can draw on their positions in the organizational hierarchy or their access to critical resources in attempts to influence framing processes [52]. For actors with less influential power positions, beliefs in whether or not they have an option to engage shape framing processes as well [1].

Culture, including values, beliefs, rituals, and knowledge, comes into play across different phases of framing processes in different ways. Both the culture in which an organization is situated and the organizational culture prevailing in an organization shape framing processes. As an example of the former, it is suggested that actors make use of externally available cultural resources (e.g., values, beliefs, rituals, knowledge) in the phase of technology concept development, which activate cultural frames. Such cultural frames guide how problems are understood - that envisioned technologies are eventually intended to solve - which in turn guides subsequent technology development [29]. This means that culture not only shapes interpretations of technology during development, implementation, or use but is also "activated" already in the earlier stage of generating ideas about technology [29]. To exemplify how the prevailing organizational culture shapes framing, the organization's pre-existing epistemic stance, referring to "an attitude that organizational actors collectively enact in pursuing knowledge," can both be a fruitful source in the reframing of technological opportunities and be a source of inertia for an organization, depending on the prevailing attitude in a particular organization [13].

\section{Suggestions for Future DT Research}

This study aimed to explore how framing theory can be applied in DT research. By reviewing framing theory in IS research, I outlined six subareas of framing theory, namely, relational, political, tactical, technological, temporal, and contextual. These subareas will now be used to suggest research questions for future DT research. While a thorough examination across levels of analysis was beyond the scope of this paper, future research can favorably approach DT on and across various levels of analysis, such as individuals, groups (including boundary-spanning actor constellations, given that organizations are situated in ecosystems), organizations, and macro-level influences on organizational life (e.g., ecosystems). In light of this paper's view on DT, recurrent meaning making, or reframings [48] as discussed here, of digital technologies and their role in digital ecosystem orchestration is integral for organizations' ability to adapt continuously in digital ecosystems.

Relational Framing. Framing theory accentuates the relational, and thereby interactional, nature of meaning making of digital technologies. DT research could explore how interactional patterns, within and across organizations, can be reconfigured to create better prerequisites for reframing, with the potential to guide organizational adaptations for improved digital ecosystem orchestration. In other words, such DT research could explore how organizations reconfigure for generative relationships, "relationship[s] that can induce changes in the way the participants see their world and act in it, and even give rise to new entities, like agents, artifacts, even institutions" [27]. In line with this paper's view on DT, such research efforts should also consider how organizations engage ecosystem actors (e.g., users, innovators, partners, etc.) in the reframing of digital technologies. It could be asked: How do organizations foster generative relationships for reframing digital technologies?

Additionally, framing theory sheds light on the impact of frames across levels. Notably, it is suggested that an established organizational frame can have a negative impact on individuals' reframing of digital technologies when returning to their natural setting [25]. In other words, the prevailing organizational frame can become a source of inertia. DT research could explore the question: How can organizations counteract the negative influence of established organizational frames on reframings of digital technologies?

Political Framing. Framing theory highlights the political character of meaning making of digital technologies, when different actors are guided by different interests or values in a technological prospect. In DT, it is likely that contests become increasingly situated in ecosystem contexts and are thereby inherently boundary spanning. For example, contests may manifest between a focal organization and other ecosystem actors or between ecosystem actors without the direct involvement of the focal organization. In addition, contests situated in digital ecosystems are likely to involve actors on a grander scale and diversity 
given the unique characteristics of digital ecosystems [23]. They may also become more frequent because digital applications and content are continuously generated anew or redesigned [8]. In this scenario, focal actors in an ecosystem with significant influence over the ecosystem dynamics [8] may face an increasing need to mobilize for, protect from or reconcile political contests within ecosystems. Likewise, less influential ecosystem actors may face similar needs if there is disagreement about how a digital ecosystem is coordinated. The following question can then be asked: How do ecosystem actors organize for and engage in the political framing of digital technologies?

Tactical Framing. Tactical framing incorporates rhetorical and organizing acts, as goal-oriented moves that actors deploy when seeking to influence change in others' frames. In DT, we can imagine such tactics to be imperative for individual actors or groups aiming to generate frame change in some particular direction (e.g., entrepreneurs, "intrapreneurs"), such as to persuade others of envisioned potentials of digital technologies (e.g., funders, boards), or stimulate shifts in practices, from improvement of extant value propositions [50] to continuous adaptation for digital ecosystem orchestration (e.g., organizational members, partners). Equally, we can imagine the importance of tactical framing for actors seeking to resist and mobilize against change in undesired directions. We know little about the particularities of tactical framing in the context of DT. Such tactical framing may involve particular challenges, including persuading organizational decision makers with primary prior experience in managing industrial organizations about the prospects of digital ecosystem orchestration, or resolving emerging tensions in incumbent organizations as they transition to DT [40]. Broadly, it could be asked: How do actors engage in tactical framing to reframe digital technologies in DT?

Tactical framing also entails methodological opportunities for DT researchers. It could provide a basis for active research engagement with emphasis on discourse as a tool for action [32], beyond engagement through design as typically applied in IS research. DT research could consider how tactical framing of digital technologies can be deployed in collaboration with practitioners in pursuit of jointly improving the understanding of DT.

Technological Framing. Framing theory recognizes that technologies play an active role in framing processes. It is proposed that technologies provide means for sustaining frames across space and time, thereby acting as a medium that diffuses frames. "The frame problem" of computational agents illustrates that this point is likewise applicable to emerging digital technologies such as AI. It posits that because algorithms (as we know today) are unable to adapt independently to new situations, developers' frames become manifested in the rule-setting in developing rule-based algorithms and in choices regarding input data during the development of ML-algorithms [39]. This outcome implies that because digital technology development is distributed in nature [33], it is likely that the frames of distributed individuals or of groups are what digital technology provides continuity for in the context of digital ecosystems. This shift to distributed technological framing can be envisioned as having implications for DT. For example, it could mean that political contests between groups are less likely to precede design decisions, which could entail both opportunities and unintended consequences. On the one hand, distributed technological framing may facilitate rapid technological changes. On the other hand, resolving an eventual contest through proactive negotiation into a shared, or at least stable, frame may have value from a democratic perspective. DT research could explore: What are the organizational opportunities and consequences of distributed technological framing in digital ecosystems?

Temporal Conditions. Temporal conditions concern how framing processes change in character over time, for example, the differences in the significance of framing in relation to technological change [24, 29] and contest progression [1, 2]. In relation to technological change, it particularly emphasizes the proactive role of framing to shape meanings of technologies during development. For digital ecosystem orchestration however, where digital technologies are potentially ever-changing and ecosystem structures continuously emerging, organizations also need capacity to engage in reactive framing, to respond to what other actors generate anew or redesign unexpectedly. The combination of proactive and reactive framing is likely imperative for DT. It could be asked: How do organizations combine proactive and reactive reframing of digital technologies for influential digital ecosystem orchestration?

Contextual Conditions. Framing theory sheds light on how actors' prior history, established power positions and the culture that surrounds and prevail in organizations impact the reframing of digital technologies. For example, it is suggested that different contextual conditions can either be a source of reframings that guide new action pathways in organizations or be a source of inertia that hinders change [13]. DT research could continue exploring what contextual conditions support the reframing of digital technologies and if/how contextual conditions that lead to inertia can be overcome, by asking: How do contextual conditions impact reframings of digital technologies, and how can those conditions with negative impact be overcome? 


\section{Conclusion and Limitations}

This paper reviewed framing theory in IS research with the aim to suggest research opportunities for DT research. Two contributions can be outlined. First, the paper contributes to the framing literature, by synthesizing theoretical contributions on the framing of digital technology / IT in organizational contexts. From a theory as process perspective, where published work is viewed as bits and pieces of a collaborative effort that gradually becomes theory [47], such "check-point" is valuable for organizing distributed theorizing efforts. Second, it contributes to the DT literature by proposing questions for future research. Thus, this paper represents an attempt to accumulate a promising theoretical foundation that can help ground future generative theorizing on DT [19].

Some significant limitations of this paper are important to recognize. First, the sample is admittedly small, as in-depth reading of a representative sample was prioritized over breadth. Second, coding was conducted by one individual only, removing the option to strengthen the analysis through intercoder reliability. Third, only papers that explicitly used framing theory were included, thereby excluding studies of relevant empirical phenomena with alternative theoretical framings. Fourth, the discussion does not discuss the potential of framing theory in relation to theoretical alternatives, where other options include, for example, sensemaking [49], sensegiving [15], sensebreaking [53], mindfulness and mindlessness [11], institutional logics [3] and social representation [14, 41]. Nonetheless, I am hopeful that the choice and scope of this review is sufficient to inspire future DT research.

\section{References}

[1] B. Azad and S. Faraj, "Making e-Government systems workable: Exploring the evolution of frames", The Journal of Strategic Information Systems, 17 (2008), pp. 75-98.

[2] B. Azad and S. Faraj, "Social power and information technology implementation: A contentious framing lens", Information Systems Journal, 21 (2011), pp. 33-61.

[3] N. Berente and Y. Yoo, "Institutional contradictions and loose coupling: Postimplementation of NASA's enterprise information system", Information Systems Research, 23 (2012), pp. 376-396.

[4] R. Bernardi, P. Constantinides and J. Nandhakumar, "Challenging dominant frames in policies for IS innovation in healthcare through rhetorical strategies", Journal of the Association for Information Systems, 18 (2017), pp. 81-112.

[5] V. Braun and V. Clarke, "Using thematic analysis in psychology", Qualitative Research in Psychology, 3 (2006), pp. $77-101$

[6] A. Burton-Jones, E. R. McLean and E. Monod, "Theoretical perspectives in IS research: From variance and process to conceptual latitude and conceptual fit", European Journal of Information Systems, 24 (2015), pp. 664-679.

[7] S. Chanias, M. D. Myers and T. Hess, "Digital transformation strategy making in pre-digital organizations: The case of a financial services provider", The Journal of Strategic Information Systems, 28 (2018), pp. 17-33.

[8] P. Constantinides, O. Henfridsson and G. G. Parker, Introduction-Platforms and Infrastructures in the Digital Age, Information Systems Research, 2018, pp. 381-400.

[9] J. P. Cornelissen and M. D. Werner, "Putting framing in perspective: A review of framing and frame analysis across the management and organizational literature", Academy of Management Annals, 8 (2014), pp. 181-235.

[10] E. Davidson, "A technological frames perspective on information tchnology and organizational change", Journal of Applied Behavioral Science, 42 (2006), pp. 23-39.

[11] S. Dernbecher and R. Beck, "The concept of mindfulness in information systems research: A multi-dimensional analysis", European Journal of Information Systems, 26 (2017), pp. 121-142.

[12] O. El Sawy, A. Malhotra, Y. Park and P. Pavlou, "Seeking the configurations of digital ecodynamics it takes three to tango", Information Systems Research, 21 (2010), pp. 835848.

[13] A.-L. Fayard, E. Gkeredakis and N. Levina, "Framing innovation opportunities while staying committed to an organizational epistemic stance", Information Systems Research, 27 (2016), pp. 302-323.

[14] U. Gal and N. Berente, "A social representations perspective on information systems implementation", Information Technology \& People, 21 (2008), pp. 133-154.

[15] D. A. Gioia and K. Chittipeddi, "Sensemaking and sensegiving in strategic change initiation", Strategic Management Journal, 12 (1991), pp. 433-448.

[16] S. J. Girod and R. Whittington, "Reconfiguration, restructuring and firm performance: Dynamic capabilities and environmental dynamism", Strategic Management Journal, 38 (2017), pp. 1121-1133.

[17] E. Goffman, Frame analysis: An essay on the organization of experience, Boston: Northeastern Univ. Press, Boston, 1986.

[18] A. Hanelt, R. Bohnsack, D. Marz and C. Antunes, "A systematic review of the literature on digital transformation: Insights and implications for strategy and organizational change", Journal of Management Studies (2020), pp. 1-39.

[19] N. R. Hassan, L. Mathiassen and P. B. Lowry, "The process of information systems theorizing as a discursive practice", Journal of Information Technology, 34 (2019), pp. 198-220.

[20] V. Higgins and M. Bryant, "Framing agri-digital governance: Industry stakeholders, technological frames and smart farming implementation", Sociologia Ruralis, 60 (2020), pp. 438-457.

[21] B. Hinings, T. Gegenhuber and R. Greenwood, "Digital innovation and transformation: An institutional perspective", Information and Organization, 28 (2018), pp. 52-61.

[22] C. Hsu, J. Huang and R. D. Galliers, "Conceptualizing the dynamics of rhetorical practice and technological frame in the context of technology diffusion and adoption", Information \& Management, 51 (2014), pp. 984-994. 
[23] F. Ivarsson and F. Svahn, "Becoming a digital ecosystem orchestrator-The Sydved case", Proceedings of the European Conference on Information Systems (2020).

[24] S. Kaplan and M. Tripsas, "Thinking about technology: Applying a cognitive lens to technical change", Research Policy, 37 (2008), pp. 790-805.

[25] C. Klos and P. Spieth, "READY, STEADY, DIGITAL?! How foresight activities do (NOT) affect individual technological frames for managerial SENSEMAKING", Technological Forecasting and Social Change, 163 (2021), p. 120428.

[26] A. Lanamäki, K. Väyrynen, S. Laari-Salmela and M. Kinnula, "Examining relational digital transformation through the unfolding of local practices of the Finnish taxi industry", The Journal of Strategic Information Systems, 29 (2020), p. 101622.

[27] D. Lane and R. Maxfield, "Strategy under complexity: Fostering generative relationships", Long Range Planning, 29 (1996), pp. 215-231.

[28] D. E. Leidner, "Review and theory symbiosis: An introspective retrospective", Journal of the Association for Information Systems, 19 (2018), pp. 552-567.

[29] P. M. Leonardi, "Innovation blindness: Culture, frames, and cross-boundary problem construction in the development of new technology concepts", Organization Science, 22 (2011), pp. 347-369.

[30] L. Li, F. Su, W. Zhang and J. Y. Mao, "Digital transformation by SME entrepreneurs: A capability perspective", Information Systems Journal (2017), pp. 1-29.

[31] H. C. Lucas Jr and J. M. Goh, "Disruptive technology: How Kodak missed the digital photography revolution", The Journal of Strategic Information Systems, 18 (2009), pp. 4655.

[32] P. Mårtensson and A. S. Lee, "Dialogical action research at Omega corporation", MIS Quarterly (2004), pp. 507-536.

[33] S. Nambisan, K. Lyytinen, A. Majchrzak and M. Song, "Digital innovation management: Reinventing innovation management research in a digital world", MIS Quarterly, 41 (2017), pp. 223-238.

[34] S. Nambisan, M. Wright and M. Feldman, "The digital transformation of innovation and entrepreneurship: Progress, challenges and key themes", Research Policy, 48 (2019), pp. 1-9.

[35] K. Olesen, "Implications of dominant technological frames over a longitudinal period", Information Systems Journal, 24 (2014), pp. 207-228.

[36] W. J. Orlikowski and D. C. Gash, "Technological frames - Making sense of information technology in organizations", ACM Transactions on Information Systems, 12 (1994), pp. 174-207.

[37] E. Pignot, D. Nicolini and M. Thompson, "Affective politics and technology buy-in: A framework of social, political and fantasmatic logics", Association for Information Systems, 2020, pp. 901-935.

[38] M. Ravishankar, "The realignment of offshoring frame disputes (OFD): An ethnographic 'cultural' analysis", European Journal of Information Systems, 24 (2015), pp. 234246.

[39] A. Salovaara, K. Lyytinen and E. Penttinen, "High reliability in digital organizing: Mindlessness, the frame problem, and digital operations", MIS Quarterly (2019), pp. 555-578.

[40] F. Svahn, L. Mathiassen and R. Lindgren, "Embracing digital innovation in incumbent firms: How Volvo cars managed competing concerns", Management Information Systems Quarterly, 41 (2017), pp. 239-253.

[41] E. Vaast, "Danger is in the eye of the beholders: Social representations of information systems security in healthcare", The Journal of Strategic Information Systems, 16 (2007), pp. 130-152.

[42] G. Vial, "Understanding digital transformation: A review and a research agenda", The Journal of Strategic Information Systems (2019), pp. 118-144.

[43] J. Vom Brocke, A. Simons, K. Riemer, B. Niehaves, R. Plattfaut and A. Cleven, "Standing on the shoulders of giants: Challenges and recommendations of literature search in information systems research", Communications of the Association for Information Systems, 37 (2015), pp. 205-224. [44] J. Wareham, P. B. Fox and J. L. Cano Giner, "Technology ecosystem governance", Organization Science, 25 (2014), pp. 1195-1215.

[45] K. S. Warner and M. Wäger, "Building dynamic capabilities for digital transformation: An ongoing process of strategic renewal", Long Range Planning, 52 (2019), pp. 326349.

[46] J. Webster and R. T. Watson, "Analyzing the past to prepare for the future: Writing a literature review", MIS Quarterly (2002), pp. xiii-xxiii.

[47] K. E. Weick, "What theory is not, theorizing is", Administrative Science Quarterly, 40 (1995), pp. 385-390.

[48] K. E. Weick and R. E. Quinn, "Organizational change and development", Annual Review of Psychology, 50 (1999), pp. 361-386.

[49] K. E. Weick, K. M. Sutcliffe and D. Obstfeld, "Organizing and the process of sensemaking", Organization Science, 16 (2005), pp. 409-421.

[50] L. Wessel, A. Baiyere, R. Ologeanu-Taddei, J. Cha and $\mathrm{T}$. Jensen, "Unpacking the difference between digital transformation and IT-enabled organizational transformation", Journal of Association of Information Systems, 22 (2021), pp. 102-129.

[51] H. Wimelius, L. Mathiassen, J. Holmström and M. Keil, "A paradoxical perspective on technology renewal in digital transformation", Information Systems Journal, 31 (2021), pp. 198-225.

[52] A. Yeow and S. K. Sia, "Negotiating "best practices" in package software implementation", Information and Organization, 18 (2008), pp. 1-28.

[53] A. Y. K. Yeow and C. E. H. Chua, "Multiparty sensemaking: A technology-vendor selection case study", Information Systems Journal, 30 (2020), pp. 334-368.

[54] Y. Yoo, O. Henfridsson and K. Lyytinen, "Research commentary - the new organizing logic of digital innovation: an agenda for information systems research", Information Systems Research, 21 (2010), pp. 724-735.

[55] B. W. Young, L. Mathiassen and E. Davidson, "Inconsistent and incongruent frames during IT-enabled change: An action research study into sales process innovation", Journal of the Association for Information Systems, 17 (2016), pp. 495-520. 